\title{
MA2-3
}

\section{Confined Modes of Two dimensional Photonic Crystal Defect Cavities with Indium Arsenide Quantum Dots}

Tomoyuki Yoshie, and Axel Scherer

Department of Electrical Engineering, MC136-93, California Institute of Techrology, Pasadena, CA 91125 , USA

telephone: 626-395-2207, facsimile: 626-683-9547, E-mail: yoshie@its.caltech.edu

Hao Chen, Diana Huffaker, and Dennis Deppe

Microelectronics Research Center, Department of Electrical and Computer Engineering,

The University of Texas at Austin, Austin, TX 78712-1084, USA

Abstract

We have fabricated and characterized two-dimensiona photonic crystal with defect cavities containing self-organized Indium Arsenide quantum dots as active material. Single defect donor modes were found to have well localized close to the single defect.

The combination ${ }^{1,2}$ of microcavities and quantum dots (QDs) has provided interesting and useful results for strong photon-atom coupling ${ }^{3}$ QDs are preferred fo such high $Q$ cavities because of the narrow linewidth emission and the atomic-like density of states. Photonic crystal nanocavities have recently attracted much attention since they are predicted to form nanocavites with small mode volumes as well as high quality factors. In this report, we investigate the optical properties of InAs QDs cavities formed by photonic crystal with a single defect or more defects.

We used MBE (molecular beam epitaxy) -grown InAs quantum dots on (001) GaAs. Three stacked InAs QDs layers were clad by $\mathrm{Al}_{0.16} \mathrm{Ga}_{0.84} \mathrm{As}$ layers on top of a $400 \mathrm{~nm} \mathrm{Al}_{0.94} \mathrm{Ga}_{0.86} \mathrm{As}$ layer. The QDs density in these samples is $3 \times 10^{10}$ dots $/ \mathrm{cm}^{2}$. A GaAs cap layer protects the top on the final layer. The cavity thickness (d) is designed to be $240 \mathrm{~nm}$. The ground state emission of QDs showed linewidths as narrow as $43 \mathrm{meV}$. The patterns to form hexagonal-arrayed photonic crystal defect cavity were defined into a $250 \mathrm{~nm}$ poly-methyl methacrylate (PMMA) resist layer spun onto the epilayers by using a field emission electron beam lithography system. The photonic crystal devices have ten layers of $\mathrm{PBG}$ surrounding a center defect. The lattice spacing (a) is lithographically controlled from $270 \mathrm{~nm}$ to $390 \mathrm{~nm}$, and the ratio of hole radius ( $\mathrm{r}$ ) to a is also changed from 0.16 to 0.4 as well. After lithography, the beam-written pattems were transferred through the active membrane by using an $\mathrm{Ar}^{+}$ion beam assisted with a $\mathrm{Cl}_{2}$ jet, and the $\mathrm{Al}_{0.94} \mathrm{Ga}_{0.06} \mathrm{As}$ layer under cavities was subsequently oxidized in steam. The $A 1_{0.94} \mathrm{Ga}_{0.06} A$ s was oxidized as far oxidized in steam. The $\mathrm{Al}_{0.94} \mathrm{Ga}_{0.06} A$ s was oxidized as far
as $3 \mu \mathrm{m}$ from the edge of photonic crystal hexagons. Figure 1 shows images taken by scanning electron

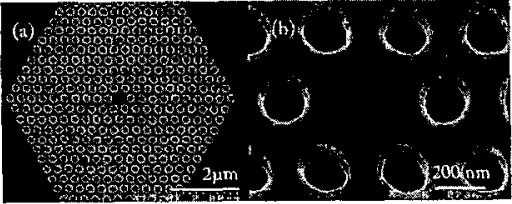

Figure 1: scanning electron microscopy images of two dimensional photonic crystal single defect cavity. microscopy for typical fully-processed photonic crystal cavities with a single defect. Light parallel to surface is confined by the photonic crystal in the plane whereas the slab structure is used to confine light to surface.

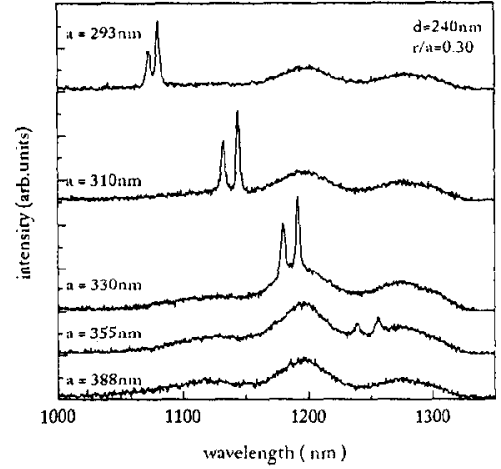

Figure 2: PL spectra for samples with different lattice periods and same $r / a$ of 0.30

The fabricated cavities were optically pumped normal to the surface by using an $830 \mathrm{~nm}$ semiconductor laser diode. The $830 \mathrm{~nm}$ light selectively can be absorbed only by the InAs QDs layers and the wetting layers. The pumping light was focused on the defect to a spot size of $2 \mu$ m diameter. Light emission extracted from the surface was detected by optical spectrum analyzer. Figure 2 shows photoluminescence (PL) spectra for photonic crystal single defect cavities with different lattice spacings and the same r/a. The cavity resonance was spacings and the same r/a. The cavity resonance was measured to exhibit a 3 to 5 nm emission linewidth. The
resonances could not be observed in spectra of photonic crystal without any defects. The spectra of the resonant modes can be tuned all over the emission range of the QDs by changing the lattice period of the photonic crystal by using lithography. The other higher order modes were totally pushed out of the $\mathrm{QD}$ emiscion spectra while photonic crystal cavities with four layers defects around center were observed to have many modes including whispering-gallery modes.

Position-dependent $\mu-P \mathrm{~L}$ measurements were conducted to roughly estimate the mode volume of the donor modes. Figure 3 shows PL spectra taken in each point described in the inset. Points labeled as $A, B, C$, and $D$ are in the photonic crystal close to the edge. Only the spectrum taken from a central point within the cavity 


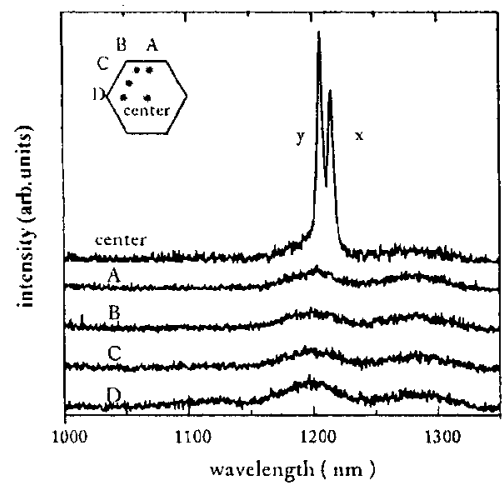

Figure 3: Position dependent PL for photonic crystal with a single defect

shows the two resonance peaks while the other points exhibit broad QDs emission. Optical pumping of points close to the edge was not coupled with the defect modes. This result indicates that the measured modes are well confined around the single defect. As seen in the figure, there exists a pair of two modes, but the mode splitting there exists a pair of two modes, but the mode splitting
of around $1 \%$ relative to the frequency in the figure possibly results from fabrication fluctuations since an accuracy of fabrication is in such a range.

The dielectric slab membrane sandwiched by air is symmetric normal to the surface and has photonic band gap for TE-like modes. However, the slab waveguides used in this work are asymmetric and nay not have a complete photonic band gap if the perturbation is complete photonic band gap if the perturbation is
relatively large as Johnson et. al. pointed out ${ }^{5}$. Our measurements showed that we could have well-confined modes around the single defect in two dimensional asymmetric slab photonic crystal cavities between air and $\mathrm{AIO}_{\mathrm{O}}$.

I J. M. Gerard, D. Barrier, J. Y. Marzin, R. Kuszelewicz, L. Manin, E. Costard, V. Thierry-Mieg, and T. Rivera, Appl. Phys. Lett. 69, 449 (1996)

2 D. G Deppe, L. A. Graham, and D. L. Huffaker, IEEE J. Quantum Electon. 35, 1502 (1999)

3 E. M. Purcell, Phys. Rev. 69, 681 (1946)

4 O. Painter, J. Vuckovic, and A. Scherer, J. Opt. Soc. An. B 16, 275 (1999)

5 S. G. Johnson, S. Fan, P. R. Villeneuve, J. D. Joannopoulos, and L. A. Kolodziejski, Phys. Rev. B $60,575 !(1999)$ 